\title{
Multidimensional Neural-Like Growing Networks - A New Type of Neural Network
}

\author{
Vitaliy Yashchenko \\ Artificial intelligence \\ Institute of Mathematical Machines and System Problems NANU, \\ IMMSP NANU \\ Kiev, Ukraine
}

\begin{abstract}
The present paper describes a new type of neural networks - multidimensional neural-like growing networks. Multidimensional neural-like growing networks are a dynamic structure, which varies depending on the external information received by receptors and the information coming from the effector area to the outside world. Multidimensional receptoreffector neural-like growing networks are supposed to store and process images of objects or situations in the subject area and manage actions through a variety of spatial representations of information, such as tactile, visual, acoustic, taste, etc. Multidimensional receptor-effector neural-like growing networks are used to design intelligent systems and electronic brains of robots. The article describes the neural-like growing networks, the basic rules for constructing the neural-like growing networks and their comparison with the normal neural networks, modeling of information flows in a human body and basic blocks and functions of electronic brains of intelligent systems and robots.
\end{abstract}

Keywords-multidimensional receptor-effector neural-like growing networks; neural networks; intelligent systems; electronic brain of robots

\section{INTRODUCTION}

In the intelligent decision making systems the knowledge processing is presented by various linguistic and logical models and some inductive and deductive constructs. These constructs, however, are not sufficient for effective modeling of complex reasoning, and they require further development. There are two approaches to their development: further development of the well-known logical constructs and search for new principles of considerations modeling, mainly due to a better understanding of thinking as a process that occurs in the brain structures. Design and development of multidimensional receptor-effector neural-like growing networks in fact present an attempt to find new principles of modeling the information processes in the human brain. It is from this point of view that the paper presents: a brief description of the neural-like growing networks; the basic rules for constructing the neural-like growing networks and their comparison with the normal neural networks; functioning of the multidimensional neural-like growing networks in modeling of information flows in the human body; a brief description of the main blocks and functions of the electronic brain of intelligent systems and robots.

\section{MULTIDIMENSIONAL NEURAL-LIKE GROWING NETWORKS}

\section{A. Neural-like growing networks}

Before proceeding to consider receptor-effector neural-like growing networks, give a brief description of neural-like growing networks, which in turn is based on multiply connected growing networks.

A multiply connected growing network (n-GN) is an acyclic graph in which the minimum number of arcs entering a node is equal to a variable coefficient $n$. where $n$ is always greater than 2. Nodes without entering arcs are called receptors; other nodes are called conceptors.

Receptors constitute the generating set of the network. Conceptors correspond to combinations of features that determine conjunctive connections among objects.

Formally, multiply connected growing networks are described by the 4-tuple: $\mathbf{S}=(\mathbf{R}, \mathbf{A}, \mathbf{D}, \mathbf{N})$, where $\mathbf{R}=\left\{r_{i}\right\}$, $i=\overline{l, n}, \quad \mathbf{A}=\left\{\mathrm{a}_{\mathrm{i}}\right\}, i=\overline{l, k}, \mathbf{D}=\left\{\mathrm{d}_{\mathrm{i}}\right\}, i=\overline{l, e} ; \mathbf{R}$ is the finite set of receptors; $\mathbf{A}$ is the finite set of conceptors; $\mathbf{D}$ is the finite set of arcs connecting receptors with conceptors and conceptors among themselves; $\mathrm{N}$ is the variable connectivity coefficient, which determines the minimum allowed number of arcs entering a conceptor.

Multiply connected growing networks are used to model neuron structures. Multiply connected growing networks in which every arc is assigned a certain weight and every node is assigned a certain excitation threshold are called neural-like growing networks (n-GN).

Neural-like growing networks are formally defined as $\mathbf{S}=(\mathbf{R}, \mathbf{A}, \mathbf{D}, \mathbf{P}, \mathbf{M}, \mathbf{N})$. Here $\mathbf{R}=\left\{\mathrm{r}_{\mathrm{i}}\right\}, i=\overline{1, n}, \mathbf{A}=\left\{\mathrm{a}_{\mathrm{i}}\right\}$, $i=\overline{l, k}, \quad \mathrm{D}=\left\{\mathrm{d}_{\mathrm{i}}\right\}, i=\overline{1, e} ; \mathbf{P}=\left\{\mathrm{P}_{\mathrm{i}}\right\}, i=\overline{1, k} \mathbf{N}=\mathrm{h}$, where $P$ is the excitation threshold of node $a ; P=f(m)>P^{\circ}$ ( $\mathrm{P}^{\circ}$ is the minimum allowed excitation threshold) given that the set of arcs $\mathbf{D}$ entering the node $a_{i}$, is assigned the set of weights $\mathbf{M}=\left\{\mathrm{m}_{\mathrm{i}},\right\}, i=\overline{1, w}$, where $\mathrm{m}_{\mathrm{i}}$, may take both positive and negative values (fig.1). 


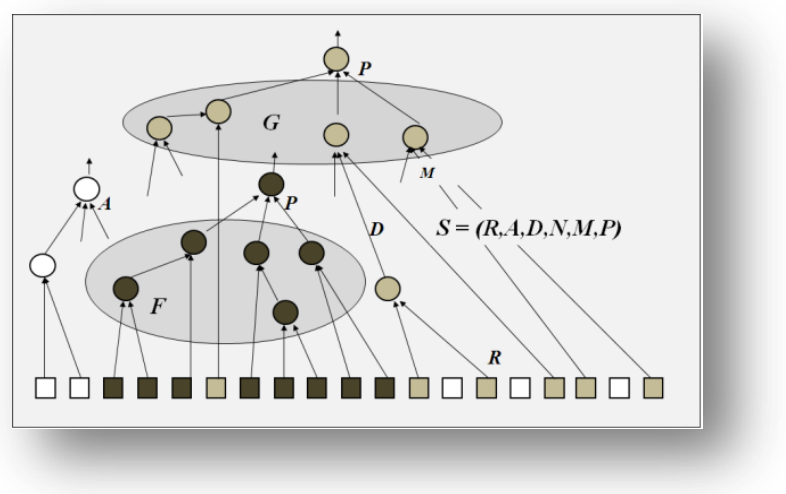

Fig. 1. Neural-like growing networks

The neural-like growing network is trained simultaneously with construction. In a network, a subset $\boldsymbol{F}$ of excited tops from the set of tops, having direct relationship with the top $a_{i}$, and subset of excited tops of the network $\boldsymbol{G}$, not having downwards relationships with other excited tops stands out. Symbols $\overline{\bar{F}}$ and $\overline{\bar{G}}$ mark the powers of subsets $\boldsymbol{F}$ and $\boldsymbol{G}$, accordingly.

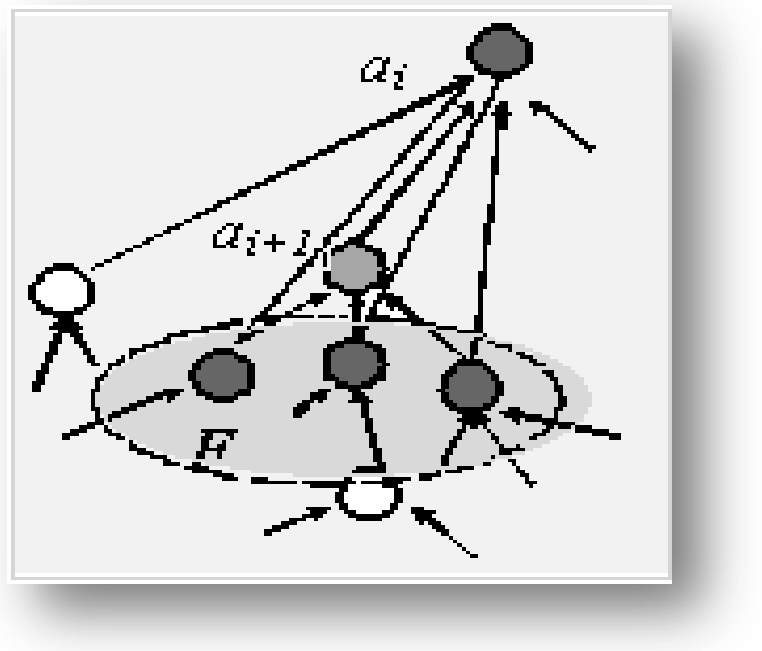

Fig. 2. Rule 1

Rule.1. If during the perception of information, a subset of tops $\boldsymbol{F}$ from the set of tops, having direct relationship with the top $a_{i}$, is excited, and $\overline{\bar{F}} \geq h$ the relationships of a top $a_{i}$ with tops from the subset $\boldsymbol{F}$ are liquidated and a new top $a_{i+1}$ joins the network, whose entries are connected with entries of all tops of the subset $\boldsymbol{F}$, and the exit of a top $a_{i+1}$ is connected with one of the inputs of a top $a_{i}$, whereas the input relationships of the top $a_{i+1}$ are assigned weighted factors $m_{i}$, corresponding to the weighted factors of liquidated relationships of the top $a_{i}$, and top $a_{i+1}$ is assigned the threshold of excitation $P_{i}$, equals $f\left(m_{i}\right)$, (function from weighted relationship factors, which fall into the top $a_{i+1}$ ).
Outcoming relationship of this top is assigned a weighted factor $m_{i}$, equal $f\left(P_{i}\right)$. Relationships, outcoming from receptors, are assigned a weighted factor, $f\left(b_{i}\right)$, function from the code of sign $b_{i}$, corresponding to a given receptor (fig.2).

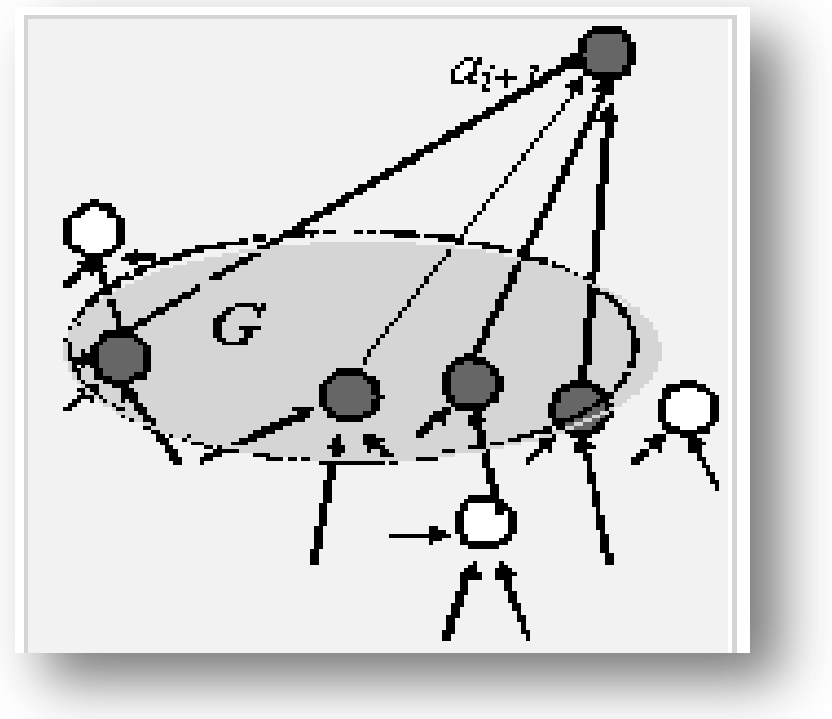

Fig. 3. Rule 2

Rule.2. If during the perception of information, a subset of tops $\boldsymbol{G}$ is excited, and $\overline{\bar{G}} \geq h$ a new associative top $a_{i+l}$, joins the network, which is connected by turning arcs with all tops of the subset $\boldsymbol{G}$. Each of turning arcs is assigned a weighted factor $m_{i}$, equal $f\left(P_{i}\right)$ of a corresponding top from the subset $\boldsymbol{G}$, and a new top $a_{i+1}$ is assigned a minimum threshold of excitement $P_{i}$, equal to the function of weighted factors $m_{i}$ of incoming arcs (fig. 3).

Information in neural-like growing networks is stored as a result of its reflecting in the structure of a network. New information input into the network causes a process of building of its structure.

\section{B. Receptor-effector neural-like growing networks}

Receptor-effector neural-like growing networks (ren-GN) are multiply connected receptor-effector growing networks in which every receptor-zone arc entering a receptor-zone node is assigned a certain weight and the node is assigned a certain excitation threshold, and also every effector-zone arc entering an effector-zone node is assigned a certain weight and the node is assigned a certain excitation threshold.

Receptor-effector neural-like growing networks are formally defined by $S=\left(R, A_{r}, D_{r}, P_{r}, M_{r} E, A_{e}, D_{e}, P_{e}\right.$, $\left.M_{e}, N\right)$.

Here $\boldsymbol{P}=\left\{P_{i}\right\}, \quad i=\overline{1, k}$, where $P$ is the excitation threshold of node $a_{i}, P=f\left(m_{i}\right)>P_{0}\left(P_{0}\right.$ is the minimum allowed excitation threshold) given that the set of $\operatorname{arcs} \boldsymbol{D}$ entering the node $a_{i}$; are assigned the set of weights $\boldsymbol{M}=\left\{m_{i}\right\}$, $i=\overline{1, w}$, where $m_{i}$, may take both positive and negative values. 


\section{Multidimensional receptor-effector neural-like growing networks}

Multidimensional receptor-effector neural-like growing networks introduced for storing and description of objects and situations in different representations, as well as for the development of control actions, stimulated by these descriptions. A plurality of interconnected two-sided cyclic graphs that describe a receptor-effector increasing neural networks in different information representations called multidimensional receptor-effector neural-like growing networks.

Multidimensional receptor-effector neural-like growing networks (mren-GN) is formally defined as:

$\mathrm{S}=\left(\mathrm{R}, \mathrm{A}_{\mathrm{r}}, \mathrm{D}_{\mathrm{r}}, \mathrm{P}_{\mathrm{r}}, \mathrm{M}_{\mathrm{r}}, \mathrm{N}_{\mathrm{r}}, \mathrm{E}, \mathrm{A}_{\mathrm{e}}, \mathrm{D}_{\mathrm{e}}, \mathrm{P}_{\mathrm{e}}, \mathrm{M}_{\mathrm{e}}, \mathrm{N}_{\mathrm{e}}\right)$; where $\mathrm{R} \supset \mathrm{R}_{\mathrm{v}}, \mathrm{R}_{\mathrm{s}}, \mathrm{R}_{\mathrm{t}} ; \mathrm{A}_{\mathrm{r}} \supset \mathrm{A}_{\mathrm{v}}, \mathrm{A}_{\mathrm{s}}, \mathrm{A}_{\mathrm{t}} ; \mathrm{D}_{\mathrm{r}} \supset \mathrm{D}_{\mathrm{v}}, \mathrm{D}_{\mathrm{s}}, \mathrm{D}_{\mathrm{t}} ; \mathrm{P}_{\mathrm{r}} \supset \mathrm{P}_{\mathrm{v}}, \mathrm{P}_{\mathrm{s}}, \mathrm{P}_{\mathrm{t}}$; $M_{r} \supset M_{v}, M_{s}, M_{t} ; N_{r} \supset N_{v}, N_{s}, N_{t} ; E \supset E_{r}, E_{d}, E_{d} ; A_{e} \supset A_{r}, A_{d 1}, A_{d 2} ;$ $\mathrm{D}_{\mathrm{e}} \supset \mathrm{D}_{\mathrm{r}}, \mathrm{D}_{\mathrm{d} 1}, \mathrm{D}_{\mathrm{d} 2} ; \quad \mathrm{P}_{\mathrm{e}} \supset \mathrm{P}_{\mathrm{r}}, \mathrm{P}_{\mathrm{d} 1}, \mathrm{P}_{\mathrm{d} 2} ; \quad \mathrm{M}_{\mathrm{e}} \supset \mathrm{M}_{\mathrm{r}}, \mathrm{M}_{\mathrm{d} 1}, \mathrm{M}_{\mathrm{d} 2}$; $\mathrm{N}_{\mathrm{e}} \supset \mathrm{N}_{\mathrm{r}}, \mathrm{N}_{\mathrm{d} 1}, \mathrm{~N}_{\mathrm{d} 2}$; here $\mathrm{R}_{\mathrm{v}}, \mathrm{R}_{\mathrm{s}}, \mathrm{R}_{\mathrm{t}}$ is a finite subset of receptors, $A_{v}, A_{s}, A_{t}$ - finite subset of neural-like elements, $D_{v}, D_{s}, D_{t}$ finite subset of arcs, $\mathrm{P}_{\mathrm{v}}, \mathrm{P}_{\mathrm{s}}, \mathrm{P}_{\mathrm{t}}$ - finite subset of excitatory thresholds of the neural-like elements of the receptor area belonging, for example, to the visual, acoustic or tactile informational dimensions, $\mathrm{N}$ - finite set of connectivity variables of the receptor area, $\mathrm{E}_{\mathrm{r}}, \mathrm{E}_{\mathrm{d} 1}, \mathrm{E}_{\mathrm{d} 2}$ - finite subset of effectors, $A_{r}, A_{d 1}, A_{d 2}$ - finite subset of neural-like elements, $\mathrm{D}_{\mathrm{r}}, \mathrm{D}_{\mathrm{d} 1}, \mathrm{D}_{\mathrm{d} 2}$ - finite subset of arcs of the effector area, $\mathrm{P}_{\mathrm{r}}, \mathrm{P}_{\mathrm{d} 1}$, $\mathrm{P}_{\mathrm{d} 2}$ - finite set of excitatory thresholds of the neural-like elements of the effector area belonging, for example, to the speech informational dimension and the action dimension. $\mathrm{N}$ finite set of connectivity variables in the effector area (fig.4).

Neural-like growing networks are a dynamic structure that changes depending on the value and the time the information gets to the receptors, as well as on the previous state of the network. The information about the objects is presented as the ensembles of excited nodes and the connections between them. Memorization of objects and situations descriptions is

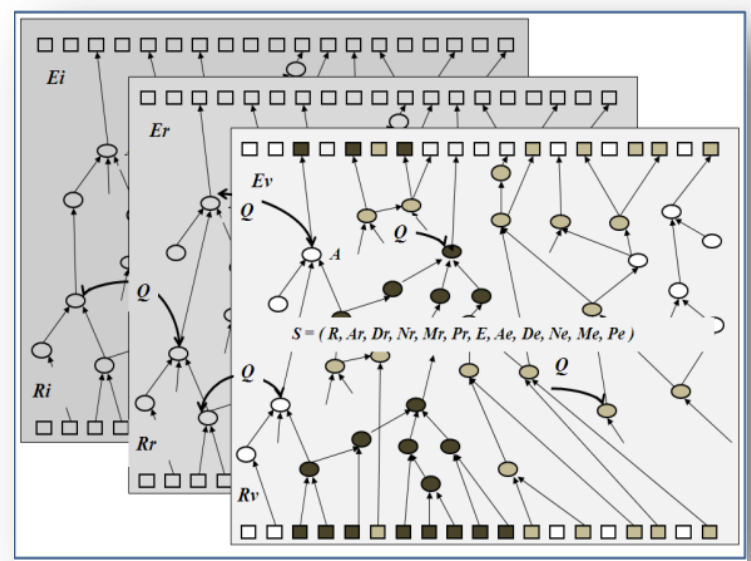

Fig. 4. Multidimensional receptor-effector neural-like growing networks accompanied by the addition of new nodes and arcs to the network when a group of receptors and neural-like elements enter into a state of excitement. There are two cases of the possibility of a neural-like element entering a state of excitement:

1) The input information is completely defined. A neurallike element enters a state of excitement if its input has received signals via all the connections.

2) The input information is not completely defined. A neural-like element enters a state of excitement if its excitatory threshold $\boldsymbol{P}=f\left(m_{i}\right)$ is greater than or equal to the minimum allowed excitatory threshold $P^{0}$. The excitation is distributed wave through the network.

Receptor-effector neural-like growing networks are a dynamic structure, which changes depending on the external information coming into the receptor field and the information generated by the effector area and transfered to the outside world.

Memorization of the external information is accompanied by the addition of new nodes and arcs to the network in the receptor zone, while the generation of information and its transfer to the outside world is accompanied by the addition of new nodes and arcs to the network in the effector area. The formation of new nodes and arcs is accompanied by the transition of an ensemble of receptors or neural-like elements, or receptors and neural-like elements of the receptor area and an ensemble of effectors or neural-like elements, or effectors and neural-like elements of the effector area into an excited state. The excitation is distributed wave through the network.

In mren-GN the information about the outside world, its objects, their states and situations describing the relationship between them, as well as the information on the actions caused by these states is saved being reflected in the network structure, and the acquisition of the new information initiates the formation of new associative nodes and links and their redistribution between the nodes that have arisen earlier, the common parts of these descriptions and actions are generalized and classified automatically. Multidimensional receptor-effector neural-like growing networks are designed to memorize and process the of images of objects or situations in the problem area and to generate the control actions with the help of various informational spatial representations, such as tactile, visual, acoustic, taste ones etc.

Now, must be compared the neural growing networks with conventional neural networks [1].

\section{Comparison of neural growing networks with conventional neural networks.}

In conventional artificial neural networks neuron model is very simple. An artificial neuron is a device with many inputs and one output. A neuron operates in accordance with the formula:

$$
x_{i}=F_{i}\left(\sum_{j=1}^{n} w_{i j} x_{j}+f_{i}-h_{i}\right)
$$

The neural network has two modes of operation; the training mode and the using mode. In the training mode, 
requires training sample and time for training (the so-called epochs of training) and difficulties arise while training and the work network in real time. The structure of each type of network is fixed and has one or two, three layers (fig.5).

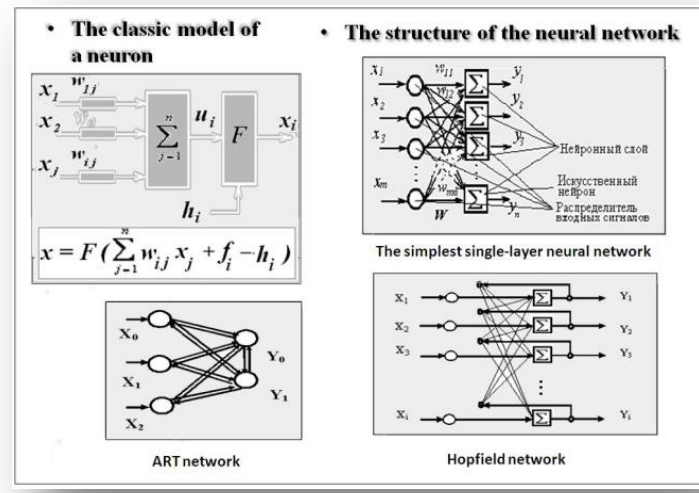

Fig. 5. The classic model of a neuron and structure of the neural networks

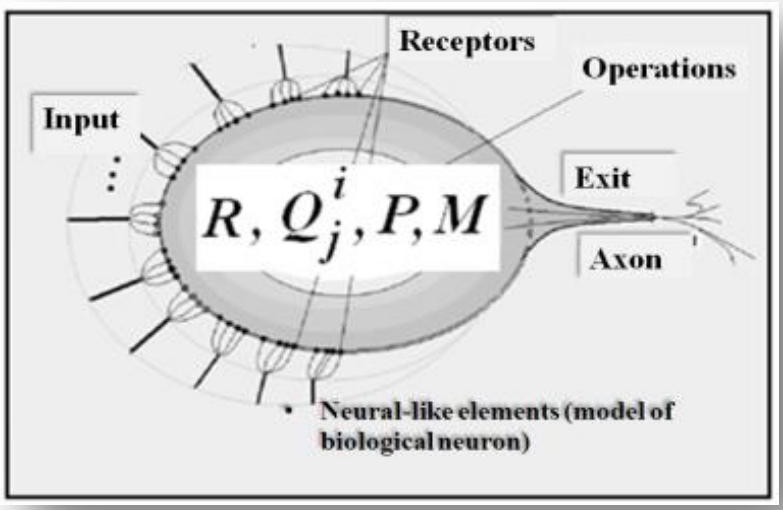

Fig. 6. The neural-like element

In neural-like growing networks of neural elements is a model of a biological neuron. Neural-like element is device with many inputs and one output. Neural-like elements determines in what respect are concepts or events, analyzes, classifies, summarizes and generates control signals to executive mechanisms (fig.6).

In neural-like growing network structure is a variable and multi-level. The number of levels (layers) is arbitrary, is generated automatically according to the input information. Information changes the network structure. Information received and processed at least three channels (video, sound, symbols). Learning occurs in real time, simultaneously with the receipt of information. The network is similar to biological neural networks.

\section{E. Constructing the multiconnection neural-like growing networks.}

The principle of constructing $\mathrm{n}-\mathrm{GN}$ (for simplicity) is considered by the example of constructing a graph of a multiconnected growing network.

\section{Example 1}

A variable coefficient is set $(\mathrm{N}=5)$.

Suppose during the time period $(\mathrm{t} 1, \mathrm{t} 2, \mathrm{t} 3, \ldots \mathrm{tk}$,$) the$ network receptors receive the following information $t 1$ (a,b,c,d); t2 - (b,c,d,e,g,h); t3 - (d,e,f); ... tk - (d,e,h) :

t1) Information $(a, b, c, d)$ comes to the receptors. Receptors 1,2,3,4 enter a state of excitement. A node (a,b,c,d) is formed. Connections are formed between the node and the receptors. The node enters a state of excitement (Fig. 7).

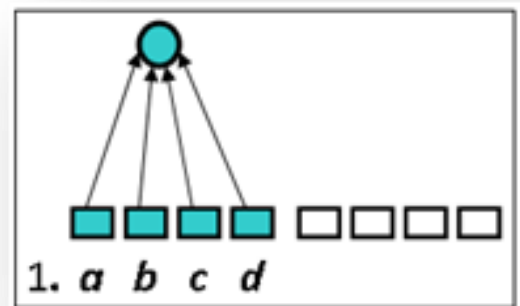

Fig. 7. The first concept is formed

t2) Information (b,c,d,e,g,h) comes to the receptors. Receptors 2,3,4,5,7,8 enter a state of excitement. Here the features $(b, c, d)$, coincide with the description of the first representation (a, b, c, d), N = 3<5, in this case the second node $(b, c, d, e, g, h)$ is formed. Connections are formed between the node and the receptors. The node enters a state of excitement (Fig. 8).

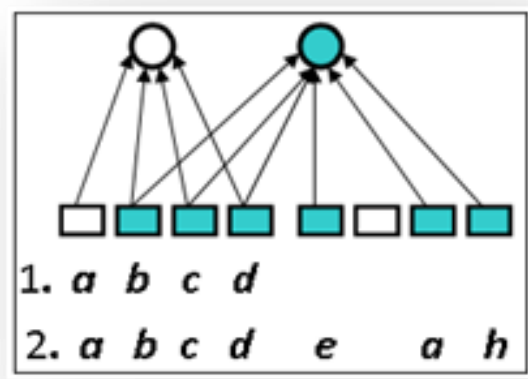

Fig. 8. The second concept is formed 
t3) Information $(d, e, f)$ comes to the receptors. Receptors 4,5,6 enter a state of excitement. Here the features $(\mathrm{d}, \mathrm{e})$, coincide with the description of the representation (b,c,d,e,g,h), N = $2<5$, in this case the second node (d,e,f) is formed. Connections are formed between the node and the receptors. The node enters a state of excitement (Fig. 9).

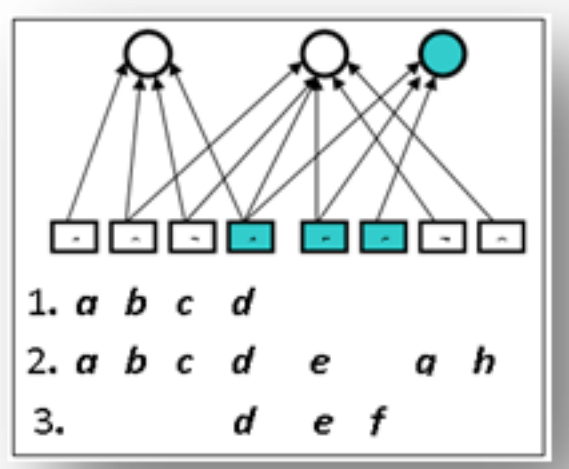

Fig. 9. The third concept is formed

tк) Information $(\mathrm{d}, \mathrm{e}, \mathrm{h})$ comes to the receptors. Receptors $4,5,8$ enter a state of excitement. Here the features $(\mathrm{d}, \mathrm{e})$, coincide with the description of the representations (b,c,d,e,g,h) and (d,e,f), N = $2<5$ and in this case the node $(\mathrm{d}, \mathrm{e}, \mathrm{h})$ is formed. Connections are formed between the node and the receptors. The node enters a state of excitement (Fig. $10)$.

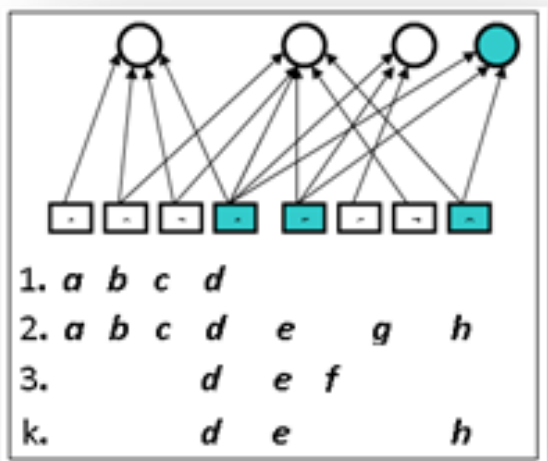

Fig. 10. $\mathrm{K}-$ the concept is formed

\section{Example 2}

A variable coefficient is set $(\mathrm{N}=3)$.

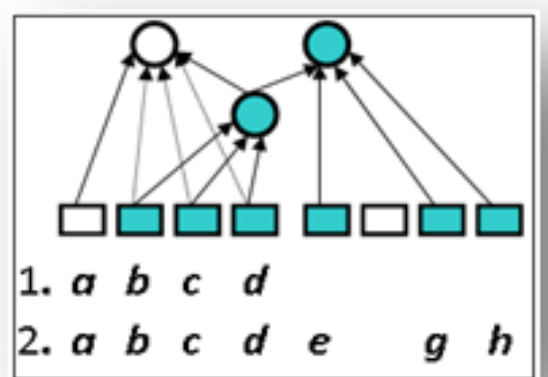

Fig. 12. The second concept is formed

Suppose during the time period $(\mathrm{t} 1, \mathrm{t} 2, \mathrm{t} 3, \ldots \mathrm{tk}$,$) the$ network receptors receive the following information $\mathrm{t} 1$ a,b,c,d; t2 - b,c,d,e,g,h; t3 - d,e,f; ... tk - d,e,h :

t1) Information $(a, b, c, d)$ comes to the receptors. Receptors $1,2,3,4$ enter a state of excitement. A node a,b,c,d is formed. Connections are formed between the node and the receptors. The node enters a state of excitement (Fig. 11).

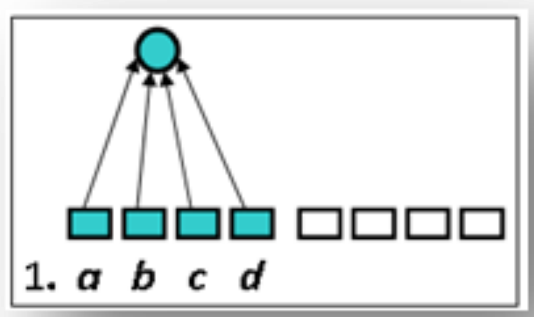

Fig. 11. The first concept is formed

t2) Information (b,c,d,e,g,h) comes to the receptors. Receptors 2,3,4,5,7,8 enter a state of excitement. A node $(\mathrm{b}, \mathrm{c}, \mathrm{d}, \mathrm{e}, \mathrm{g}, \mathrm{h})$ is formed. Connections are formed between the node and the receptors. Here the features (b, c, d), coincide with the description of the first representation $(a, b, c, d), N=$ 3 and a node (b,c,d) is formed. The connections 2,3,4 of the receptors with the node $(\mathrm{a}, \mathrm{b}, \mathrm{c}, \mathrm{d})$ are eliminated. Connections are formed between the node (b,c,d) and the nodes (a, b, c, d) and $(\mathrm{b}, \mathrm{c}, \mathrm{d}, \mathrm{e}, \mathrm{g}, \mathrm{h})$. The nodes enter a state of excitement (Fig. 12).

t3) Information (d,e,f) comes to the receptors. Receptors 4,5,6 8 enter a state of excitement. A node $(\mathrm{d}, \mathrm{e}, \mathrm{f})$ is formed. Connections are formed between the node and the receptors. The node enters a state of excitement (Fig. 13). 


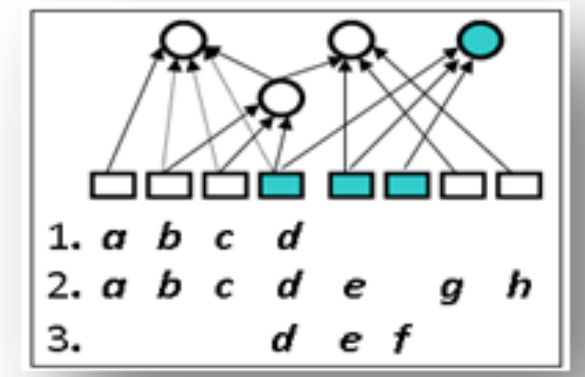

Fig. 13. The third concept is formed

tк) Information $(\mathrm{d}, \mathrm{e}, \mathrm{h})$ comes to the receptors. Receptors $4,5,8$ enter a state of excitement. Here the features $(\mathrm{d}, \mathrm{e})$, coincide with the description of the representation $(\mathrm{d}, \mathrm{e}, \mathrm{f}), \mathrm{N}=$ $2<3$ and in this case the node $(\mathrm{d}, \mathrm{e}, \mathrm{h})$ is formed. Connections are formed between the node and the receptors. The node enters a state of excitement (Fig. 14).

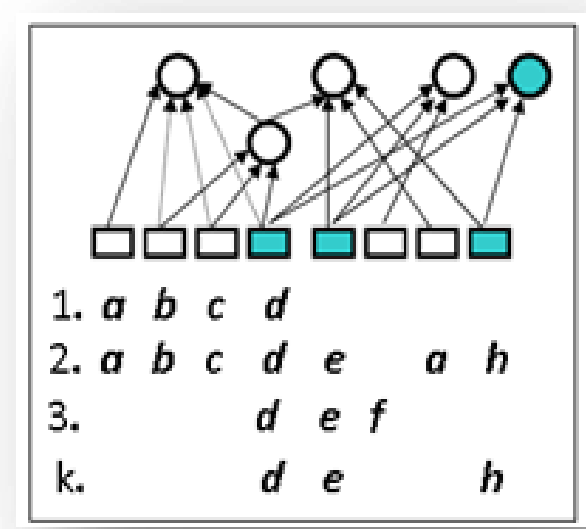

Fig. 14. $\mathrm{K}$ - the concept is formed

tK +1$)$ Information (b,c,d,e,f,g,h) comes to the receptors. Receptors 2,3,4,5,6,7,8 and nodes (b,c,d), (b,c,d,e,g,h), (d,e,f), $(\mathrm{d}, \mathrm{e}, \mathrm{h})$ enter a state of excitement. The node $(\mathrm{b}, \mathrm{c}, \mathrm{d}, \mathrm{e}, \mathrm{f}, \mathrm{g}, \mathrm{h})$ is formed. Connections between the node $(b, c, d, e, f, g, h)$ and nodes (b,c,d,e,g,h), (d,e,f), (d,e,h) are formed. The node (b,c,d,e,f,g,h) enters a state of excitement (Fig. 15).

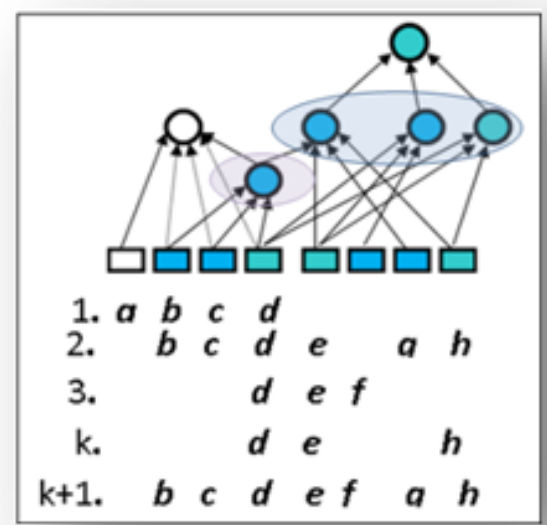

Fig. 15. $\mathrm{K}+1-$ the concept is formed

Fig.15 shows an example of the information consolidation.

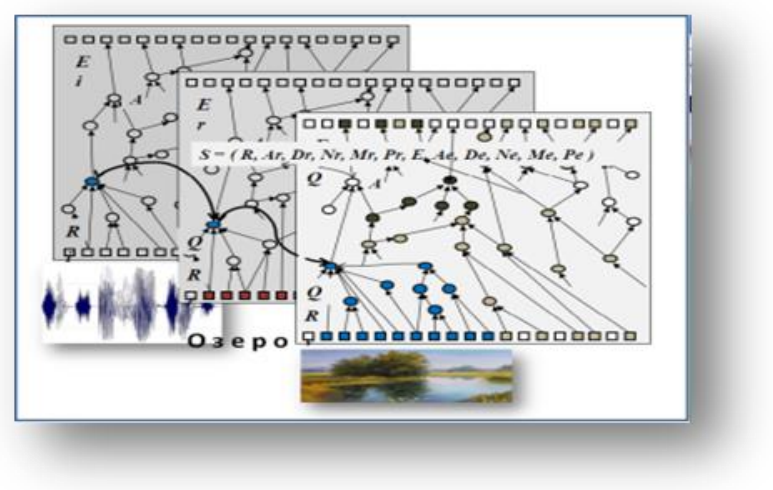

Fig. 16. The multi-dimensional network in which the concept of "lake" is represented in three dimensions (sound, symbol, vidio)

Fig.16 shows an example of the multidimensional neurallike growing network, where the concept of "lake" is represented in three dimensions (sound, symbol, video).

When the concept of "lake" is activated in one dimension, for example, in written form, the neural-like elements related to this concept get excited in the audio and visual dimensions at the same time. And the system can provide a description of this concept both in visual and sound forms. If the term "lake" is activated, for example, in visual form, the system can provide a description of this concept in written and sound forms.

Thus, the concepts descriptions are stored in the network in different representations. In addition, the information coming into the receptors zone is classified, structured and combined. 


\section{MODELING OF INFORMATION PROCESSES IN THE HUMAN BRAIN}

\section{A. Information flows in the human body}

The hypothetical scheme of information flow in man, is shown in Fig. 17. Under this scheme, the external information acts on receptors that generate nerve impulses. Combinations of stimuli are processed in the sensory area where the information flow is significantly reduced. Combinations of stimuli are combined into classes and subclasses. The information flow reaches the upper levels of the sensory area the area of the subconscious information processing.

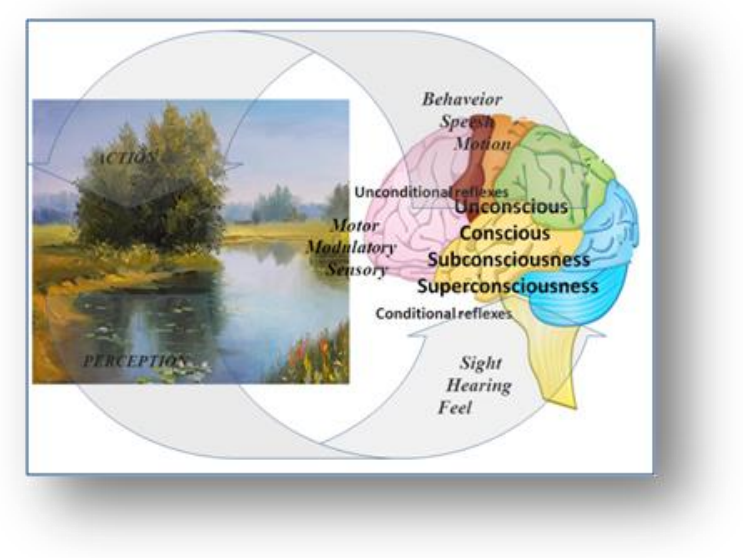

Fig. 17. The hypothetical scheme of information flow in man

Let us consider the information flows in man.

For example, an artist sees a beautiful forest lake. In this case, his brain receives external information "beautiful lake view" and a thought "beautiful landscape" occurs, the inside information - motivation "desire to reproduce it on the canvas" and accumulated as motives orders to "remember" or "make a pencil drawing" if there is external information "availability of a pencil and paper," or "draw a sketch" if there is external information " availability of paint and canvas," "availability of time" and any other information related to this situation. In accordance with this information by forming connections an order for the motor area which means the command "Draw" is produced. This command in the motor area is divided into a sequence of individual actions "set the canvas", "take a brush," "take the paint," etc. When the sketch is made, the inside information flow and motivation stop, the excitement does not reach the motor area, and the process is terminated.

Five main functions in the scheme of information flow:

perception - information from the outside world comes through the organs of perception into the area of storage of unconditioned reflexes - primary automatisms and formation of conditioned reflexes, generalization and memorization;

modulation - adjusts the level of excitation of nerve cells and performs selective modulation of a specific function;

action - information affects the outside world through the effector organs; unconscious - external information, bypassing the consciousness, causes the reaction on the outside world (unconditioned reflex);

conscious - external information through the consciousness and motivation causes the reaction on the outside world. This includes speaking through one's thoughts, micromotions, conscious actions - actions taken under control (attention), conditioned reflex.

Additional features and information processing on an unconscious level.

Unconscious - contains everything that has been previously experienced and can again be experienced consciously under certain conditions. These are automatic skills, norms of human behavior, motivation and conflicts, repressed from the conscious. It is at this level that the recollection process takes place. For example, we can not remember the name of the famous composer, writer or artist, but after some time, while our brain is already busy processing other information, we remember the name. Obviously, the search for the name is performed simultaneously with the new task on a subconscious level, and when the name is found, the process is activated and breakes through to the conscious level. Due to this parallel or simultaneous brain activity, a huge amount of information can be processed simultaneously.

Superconscious or intuition is characterized by a hidden brain activity. "Superconscious is the source of new information, hypotheses and discoveries. Its neurophysiological basis is the ransformation of memory traces and generation of new combinations, new time connections, creation of analogies"[3].

The brain is constantly looking for new combinations of all the phenomena experienced by man. Combinations are generated in the subconscious automatically, and the most active ones get through onto the conscious level.

In accordance with these features, the interaction of various organs of a living organism in a changing environment and the behavior of all the organism in the environment is determined by conditioned and unconditioned reflexes.

Unconditioned reflexes correspond to innate properties of a nervous system, and reflexes are formed when various stimuli occur simultaneously in several recurring cases. Unconditioned reflexes are formed at constant not depending on time connections between receptors and effectors, which determine the response to each stimulus. Conditioned reflexes determine the body's adaptation to changing environment.

A generation of conditioned reflexes involves creating new connections. A conditioned reflex is a basis for training a living organism and its selftraining [2].

Modeling information processes that occur in the cerebral cortex requires a structure that apart from perceiving, comparing, classifying and analyzing information can generate signals that ensure certain actions caused by the outside and motivational (inside) information. In other words, we first need to model the mechanisms of conditioned and unconditioned reflexes and then the mechanisms of conscious 
activity, providing maximum parallelism of information processing.

This structure thus should include the function of action, because knowledge is necessary for performing actions, which in turn will lead to a knowledge increase [3]. These functions are modeled in multidimensional receptor-effector neural-like growing networks.

\section{B. Information flows in the intelligent system}

The hypothetical scheme of information flow in the intelligent system is shown in Fig. 18. Under this scheme, the outside information acts on receptors that connect to neural elements. Excited elements are processed in the sensory area. Combinations of stimuli are combined into classes and subclasses. The information flow reaches the upper levels, i.e. the area of information subconscious processing.

The mren-GN structure also includes conditional and unconditional reflex zones, as well as the areas that accumulate knowledge and motivation. This mren-GN structural division is relative, since the elements are randomly distributed in different areas throughout the network.

Let us consider the information flow in the system.

For example, the system "sees" the forest lake. Receptor of the system receive the outside information, the unconditioned reflexes "desire to remember" or "draw" get excited. The command neuron generates the commands "remember the

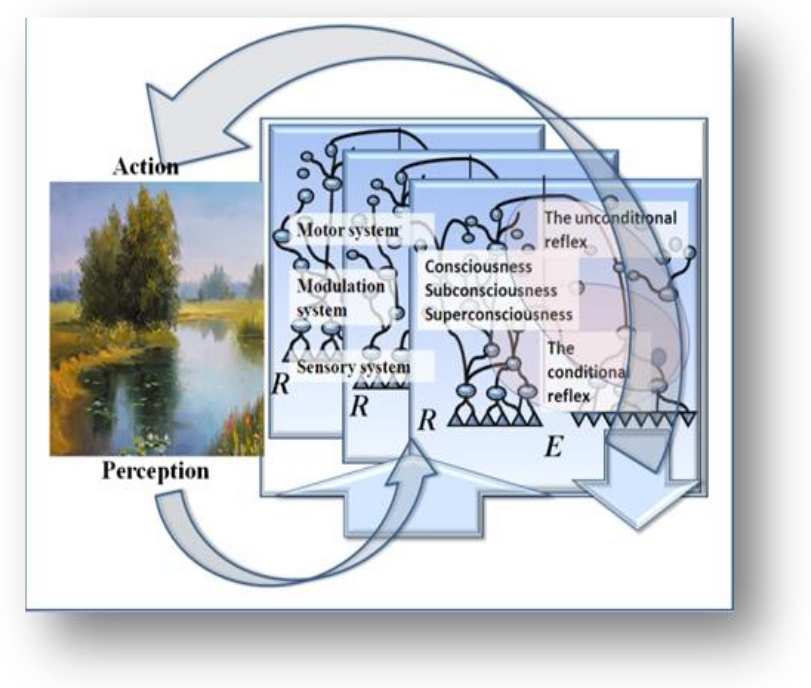

Fig. 18. The hypothetical scheme of information flow in intelligent system

scene", "draw". If there is the outside information "a pencil and paper available," - the command "draw a sketch a pencil". If there is the outside information indicating "paint and canvas available"- the command "paint a sketch." If there is the information "time sufficient", the modeling neuron sends a signal to allow a command neuron "paint a sketch" and turn off a signal for the command "draw a sketch a pencil". Thus, there are connections established in a receptor zone according to the available information and commands generated in the motor zone. In the motor zone, these commands split into a number of separate operations, "take a brush," "take the canvas", "take the paint" and so on. When the painting is completed, the inside information and motivation flow stops, no further stimuli are transmitted to the motor zone, and the whole process stops.

Five main functions in the scheme of information flow:

Perception - information from the outside world comes to the receptors area (the zone of storage of unconditioned reflexes and formation of conditioned reflexes), is generalized, stored and processed in accordance with the network formation rules.

Action - information from the receptor zone classifies and summarizes the target situation in the effector zone, strengthening or weakening the excitement of the related neural elements. Thus, in the effector area the signals that control the effector organs interacting with the outside world are generated.

Unconscious action - outside information acts on the outside world through the zone of unconditioned reflexes.

Conscious action - outside information acts on the outside world through the zone of conditioned reflexes and knowledge.

Unconditioned reflexes are innate, while the conditioned ones are acquired.

Subconscious - parallel search for several target situations in the unconditional reflex area on an unconscious level.

Superconscious - analysis and synthesis of information in different information spaces (on a subconscious level). The search for new combinations (accidentally, by analogy, in accordance with the rules) and the formation of new connections and nodes.

\section{SOME ELECTRONIC BRAIN IMPLEMENTATION ISSUES}

\section{A. Functional brain systems}

Analysis of the artificial intelligence studies shows the need to regard these studies in a system based on a common universal concept or idea, which dates back to its functional prototype - man. The classic version of the integrative activity of the human brain is known to be presented as the interaction of three main functional blocks: 1) sensory information input and processing unit - sensory systems (analyzers); 2) modulating, nervous system activating unit - brain system (limbic-reticular system) modulation; 3) programming, activating and behavioral acts controlling unit - motor systems (motion analyzer). These brain functional systems present one of the most interesting properties of the human brain - the ability to respond to an infinite number of the environmental states with a finite number of reactions.

This property might have allowed people to reach the highest form of existence of living matter, which is expressed in the ability to think, actively reflect the objective world in the form of images, concepts, judgments, and so on. Based on these provisions, which have become fundamental in the artificial intelligence theory [4,5], a set of intelligent systems "Diagnosis of Deseases", "Dialogue" and "Pattern 
Recognition" was designed. The result is a virtual personality, artificial robot VITROM, which perceives knowledge, thinks, learns, recognizes a human being having "seen" the image of his face in the video camera.

\section{B. Virtual artificial personality - robot VITROM}

The VITROM system performs in several modes: 1. Objects recognition in real time. Absence of the object for recognition (no signal in the mren-GS visible zone). No signal - unconditioned reflex circuits switch on - "attention", "search", "call". Motivation - "calls an object", "go to it." When the object appears (Fig. 19), the robot tries to recognize

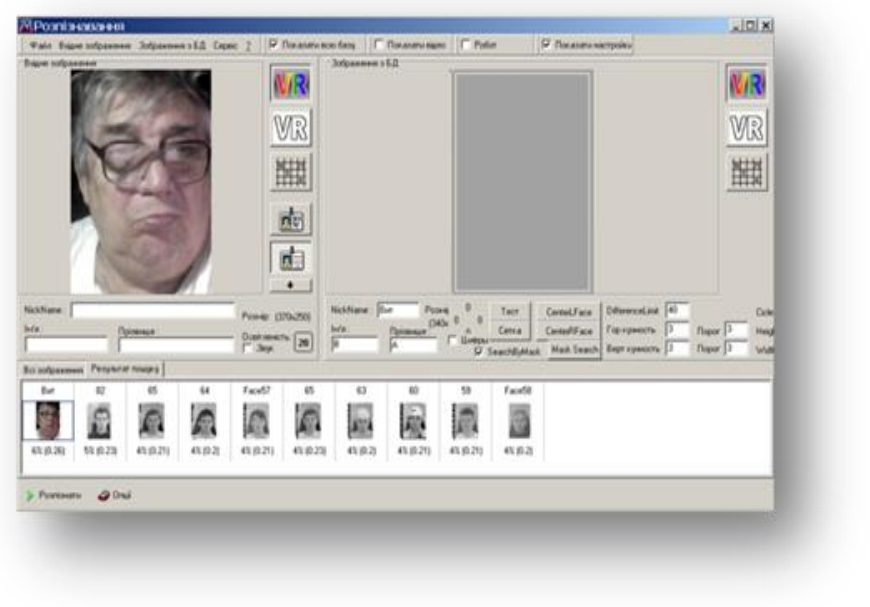

Fig. 19. The object is in the sight of the robot

the object. If the object is in sight but at a considerable distance. (ensembles of neural elements corresponding to the unconditioned reflexes "examine", "approach" in the receptor zone get excited). Robot invites you to come closer (sound field signals are generated in the effector area) and tries to recognize the object (the video signals analysis neural

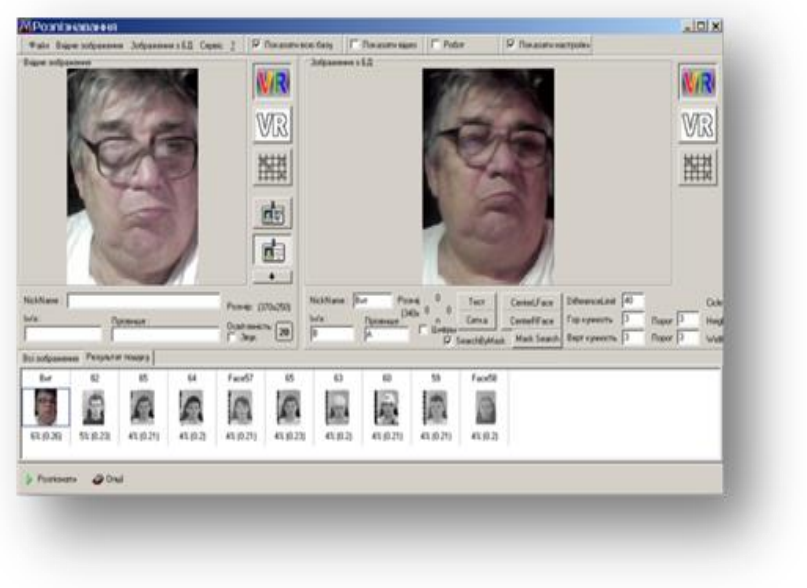

Fig. 20. The robot recognizes object

elements get excited). The object is being detected Figure 20. 2. Recognition of images from a file
The memory of the robot has memorized 1,000 images from the image database file "Yale FaceIMAGES_Data». Robot detects all the images. The recognition of images from the image database file «Yale FaceIMAGES_Data» is shown in Fig. 21.

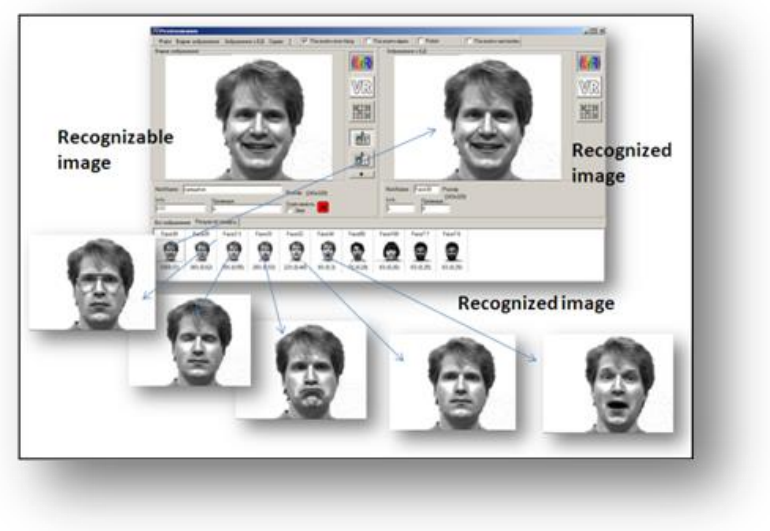

Fig. 21. The robot recognizes of image from the image database file «Yale FaceIMAGES _Data»

\section{StruCtural Diagram oF the Electronic Brain oF ROBOTS}

Structural diagram of the electronic brain of a robot is homogeneous, multi-connected, multidimensional associative active neural-like growing matrix structure (Fig. 22), it consists of the following devices: 1. perception of information (PI); 2. the set of homogeneous, multi-connected, multidimensional associative, active, neural-like matrices (HMMAASNM) - short- and long-term associative memory; 3. modulation matrices $(\mathrm{mm})$; 4. the set of homogeneous, multi-connected, multidimensional associative, active, neurallike matrices (HMMAASNM) - action block; 5. control unit (CU); 6. Power unit (PU); 7. Drives. (A). In accordance with the artificial intelligence theory in the brain of a robot $(1,2$ blocks) information about the outside world is analyzed, processed and stored in the multidimensional, active, associative memory (2). New information causes the activation of new neural-like elements and their redistribution between the elements that have arisen earlier. Information about the necessary actions caused by these conditions is analyzed (3), action neural-like elements get excited (4), necessary actions are carried out (7) and the main functions of natural intelligence (perception, analysis, synthesis, selection and storage of visual, symbolic, etc, information, communication, thinking and logical reasoning) are implemented.

\section{CONCLUSION}

The proposed concept allows us to combine the physical and the virtual worlds of intelligent systems and robots and has a universal nature. This approach provides a new framework for the development and mass production of advanced viable robots, intelligent control systems and computer systems with active associative neural-like architecture that is fundamentally different from the the von 
Neumann's one. This architecture is by low cost, small size and low power consumption. Homogeneity of the structure involves the simultaneous execution of operations over the entire active structure, wide-scale parallel and ultrafast system. Unlike the existing technologies, along with an increase in the memory size of the system the speed is increased due to the fact that data processing is carried out simultaneously in all the neural-like elements. Despite the increase in the memory size, the processing time is constant and equal to the processing time of one neural element. Neural-like growing networks were developed in 1994 -1999. Over 15 years they were tested and their von Neumann architecture based models successfully function. On the basis of these networks the artificial intelligence theory was developed. The "Artificial Intelligence" book was written, it gives the description of the methodology of the artificial intelligence development. One of the most famous British futurologists Ian Pearson states: 2015. In everyday life, home robots are commonly used as service workers. In developed countries, their number would compare with the number of cars. At the same time artificial intelligence systems are actively developing; 2018. Artificial

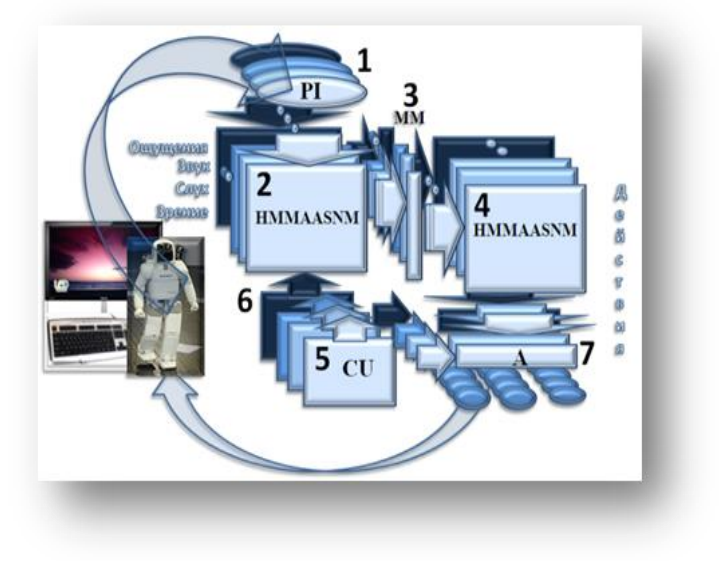

Fig. 22. Структурная схема электронного мозга робота
Intelligence would receive the Nobel Prize; 2020. Electronic forms of life would have constitutional rights; 2025. In developing countries, there would be more robots than humans; 2030. Robots, both physically and mentally, would surpass humans. And it seems that Pearson was not mistaken. 2015, there are already robots that can dance, sing, play musical instruments, serve customers, work as waiters, advertise and sell products. At the moment, the developers of robotic systems see robots as people's mechanical helpers that are able to perform various operations inherent in their program and respond to the environment.

The artificial intelligence theory allows us to get close to the implementation of robots with the intelligence equal to the human one. Here the task is to privide the robot with the electronic "brain", which would allow the robot to adapt to the environment, to acquire knowledge and make decisions leading to the achievement of the target, i.e. perform such operations that have not been programmed at the stage of robot development. In the future, the hardware implementation of this technology will enable a new type of intelligent media, thinking and sensing machines and robots.

The main advantage of the new technology, that has no technical analogues, is its hardware implementation. Unfortunately, this solution is not possible in Ukraine today.

\section{REFERENCES}

[1] V.A. Yashchenko, Receptor-effector Neural-like Growing Network efficient tool for building intelligence systems // Proc. of the second international conference on information fusion, (California, July 6-8 1999). - Sunnyvale Hilton Inn, Sunnyvale, California, USA, 1999. -Vol. II. - P. $1113-1118$.

[2] E.N.Sokolov, The principle of vector coding in psychophysiology // Moscow University Messenger. Series 14: Psychology. -1995.- № 4.P.3- 13 .

[3] N. N. Danilov and A. L. Krylova, Physiology of Higher Nervous Activity [in Russian], Izd. MGU, Moscow (1989).

[4] Yashchenko V. Artificial intelligence theory (Basic concepts) // Science and Information Conference 2014 August 27-29, 2014 | London, UK pp 473- 480

[5] https://www.google.com.ua/search?q=Yashchenko+V.+A.+Neurallike+growing+network 